\title{
Laser chirping in inverse Compton sources at high electron beam energies and high laser intensities
}

\author{
B. Terzićœ, ${ }^{1, *}$ J. McKaig $\odot,{ }^{2}$ E. Johnson $\odot,{ }^{1}$ T. Dharanikota $\odot,{ }^{3}$ and G. A. Krafft ${ }^{1,4}$ \\ ${ }^{1}$ Department of Physics, Center for Accelerator Science, Old Dominion University, \\ Norfolk, Virginia 23529, USA \\ ${ }^{2}$ George Mason University, Fairfax, Virginia 22030, USA \\ ${ }^{3}$ University of Chicago, Chicago, Illinois 60637, USA \\ ${ }^{4}$ Thomas Jefferson National Accelerator Facility, Newport News, Virginia 23606, USA
}

(Received 4 June 2021; accepted 18 August 2021; published 29 September 2021)

\begin{abstract}
The onset of nonlinear effects, such as ponderomotive broadening, increases the radiation bandwidth and thereby places a stringent limitation on the laser intensity used in inverse Compton sources. Recently, we have shown that a judicious longitudinal laser frequency modulation ("chirping") can perfectly compensate for this ponderomotive broadening and restore the narrow band property of scattered radiation in the Thomson regime, when electron recoil during the collision with the laser can be neglected. Consequently, using QED, the laser chirping has been extended to the Compton regime, where electron recoil is properly accounted for. Here we present a new, semiclassical model for computation of scattered spectra in the Compton regime. We also derive a comprehensive generalization of the expressions for chirping prescription for linearly polarized laser pulses in 1D plane-wave approximation with arbitrary shapes and arbitrary scattering angle in the Compton regime. We use these new expressions to show that the higherorder harmonics in sources with high laser fields and high electron beam energies (nonlinear Compton regime) will be nonlinearly redshifted when compared to those with lower beam energies (Thomson regime). The chirping prescription will act to correct ponderomotive broadening in very high harmonics.
\end{abstract}

DOI: 10.1103/PhysRevAccelBeams.24.094401

\section{INTRODUCTION}

Inverse Compton sources (ICS) are becoming increasingly appealing sources of radiation in regimes either not covered by other high-brilliance sources (free electron lasers, synchrotron light sources) or when compactness and portability are desired, as is the case for many applications in hospitals, labs, universities, homeland security and other fields [1]. Owing to their superior spectral densities, ICS have become preferred $\gamma$-ray sources for uses in nuclear photonics, photonuclear and fundamental physics [2-4]. In order to optimize the performance of these ICS sources, it is essential to develop an accurate analytic formalism and associated computational tools in all regimes of operation.

Recently, we developed a novel and quite general analysis of the interaction of a high-field frequencymodulated (chirped) 1D plane-wave high-intensity laser and a relativistic electron, which resulted in the perfect

\footnotetext{
*bterzic@odu.edu

Published by the American Physical Society under the terms of the Creative Commons Attribution 4.0 International license. Further distribution of this work must maintain attribution to the author(s) and the published article's title, journal citation, and DOI.
}

recovery of the narrow band property for backscattered radiation [5]. This finding was spurred by an empirical realization that frequency modulation (FM) improves spectral properties of the emitted radiation reported in Ref. [6]. We then refined the laser model by representing it as a $3 \mathrm{D}$ pulse [7]. The key idea behind laser chirping is minimizing the spectral width in the lab frame by arranging the frequency in the beam frame to emit radiation Doppler shifted back to a constant frequency in the lab frame. The resulting judicious FM of the incoming laser pulse entirely eliminates the ponderomotive broadening from the scattered radiation spectrum [5]. The optimal FM reported in these studies was derived for a special case of backscattered radiation produced in a collision of a laser beam and a single, on-axis electron. A consequent study used QED to derive the optimal FM for a Gaussian laser pulse in the Compton regime, when electron recoil is not negligible, and showed that it is dependent on electron energy and the scattering angle [8].

In this paper, we use a semiclassical approach to derive a comprehensive prescription for optimal FM at high electron energy and high laser intensity, and for an arbitrary laser pulse shape. We use the new results to study the combined impact on the scattered spectra of the two nonlinear effects: one due to high laser intensities and the other due to high electron beam energies. 


\section{REGIMES OF OPERATIONS OF INVERSE COMPTON SOURCES}

The fundamental elements of the Compton source are an electron beam and a laser, which interact to produce $\mathrm{x}$ rays or $\gamma$ rays. As a photon is scattered by an electron beam, its energy $E_{\mathrm{L}}$ is amplified by the interaction through a double Doppler shift (one shift into the frame of the electron, and the other out) to yield scattered energy [9]:

$$
E^{\prime}=\frac{4 \gamma^{2} E_{\mathrm{L}}}{1+X} \frac{\left(1+\beta_{\mathrm{CM}} \cos \theta_{\mathrm{CM}}\right)}{2},
$$

where $E_{\mathrm{L}}=2 \pi c \hbar / \lambda$ is the average energy of a photon in the laser pulse, $\lambda$ its wavelength, $c$ the speed of light. $\gamma=E_{e} /\left(m_{e} c^{2}\right)$ is the relativistic factor of the electron beam with energy $E_{e}, m_{e}$ is the mass of the electron, $\theta_{\mathrm{CM}}$ is the scattering angle in the center of mass frame, $\beta_{\mathrm{CM}}=\sqrt{1-1 / \gamma_{\mathrm{CM}}^{2}}, \gamma_{\mathrm{CM}}=\gamma / \sqrt{1+X}$, and $X$ the parameter quantifying the recoil of the electron in the collision:

$$
X=\frac{4 E_{e} E_{\mathrm{L}}}{\left(m_{e} c^{2}\right)^{2}} .
$$

Depending on the properties of these two fundamental elements of the Compton sources-the energy of an electron beam and the intensity of a laser, there are several regimes of operations.

As the energy of the electron beam increases, there are two regimes: (i) Thomson regime at low electron beam energies, where the photon energy in the rest frame of an electron is much smaller than the rest energy of an electron $(X \ll 1)$, so that the electron recoil can be neglected; (ii) Compton regime at high electron beam energies, where the photon energy in the rest frame of an electron is similar to or larger than that of an electron $(X \gg 0)$. At these energies, the electron recoil must be accounted for. As shown above, the importance of the electron recoil is that it shifts down the scattered energy by $1 /(1+X)$ [9-11].

As the intensity (power per unit area) of the laser increases, there are two regimes: (i) Linear regime at low laser intensities; (ii) Nonlinear regime at high laser intensities. The onset of nonlinearity is gradual and is best quantified by the increase in the amplitude $\left(a_{0}\right)$ of the normalized vector potential representing the laser $\tilde{A}(\xi)=e A(\xi) /\left(m_{e} c\right)=a(\xi) \cos (2 \pi \xi / \lambda)$, where $a(\xi)$ is the envelope of the laser pulse, $\xi=z+c t$ is the coordinate along the laser pulse, and $e$ the magnitude of the electron charge. Increasing the laser intensity changes the longitudinal velocity of the electrons during their collision, which leads to considerable broadening in the scattered radiation spectra $[12,13]$, as well as the nonlinear redshift in the peak frequency by a factor of $1 /\left(1+a_{0}^{2} / 2\right)$. The effects of the ponderomotive broadening are so deleterious that most ICS either remain at low laser intensities or pay a steep price to operate at a small fraction of the physically possible peak spectral output. This problem has been solved by introducing a judicious FM of the incident laser pulse to entirely eliminate the nonlinear ponderomotive broadening and allow access to high laser intensities without penalties. Earlier work provided derivation of optimal FM for specific regimes of operations of ICS and specific laser pulse shapes: backscattering off an arbitrary laser pulse in Thomson regime [5] and Gaussian laser pulse in the Compton regime [8]. It is therefore of key importance to provide a complete and most general derivation of the optimal FM for all regimes of operation and all laser pulse shapes, so as to enable a more efficient proliferation of this transformative technique.

\section{MODEL FOR COMPUTING RADIATION SPECTRA IN THE NONLINEAR COMPTON REGIME}

The general formula for linear Compton scattering is given by [10]

$$
\frac{d^{2} E}{d \omega^{\prime} d \Omega}=\frac{\epsilon_{0}}{2 \pi c}|\tilde{E}(k)|^{2} \frac{d \sigma}{d \Omega}\left[\frac{\omega^{\prime}}{\omega} \frac{d \omega}{d \omega^{\prime}}\right],
$$

where $\tilde{E}(k)$ is lab frame spatial Fourier transform of the incident photon field

$$
\tilde{E}(k, t)=\int_{-\infty}^{\infty} E(z, t) e^{-i k z} d z,
$$

and $k=\omega\left(\omega^{\prime}\right) / c$ is the wave number in the Compton regime. To get the number of photons scattered, $d^{2} N /\left(d \omega^{\prime} d \Omega\right)$, simply divide $d^{2} E /\left(d \omega^{\prime} d \Omega\right)$ by $\hbar \omega^{\prime}$. The relationship between the incoming laser frequency $\omega$ and the scattered radiation frequency $\omega^{\prime}$, accounting for electron recoil in the Compton regime, is given by

$$
\begin{gathered}
\omega\left(\omega^{\prime}\right)=\frac{\omega^{\prime}(1-\beta \cos \theta)}{1+\beta-\frac{\hbar \omega^{\prime}}{\gamma m_{e} c^{2}}(1+\cos \theta)}, \\
\omega^{\prime}(\omega)=\frac{\omega(1+\beta)}{1-\beta \cos \theta+\frac{\hbar \omega}{\gamma m_{e} c^{2}}(1+\cos \theta)} .
\end{gathered}
$$

The equivalent expressions for the Thomson regime are recovered from Eqs. (5) and (6) by setting $\hbar=0$ :

$$
\begin{gathered}
\omega_{\mathrm{T}}\left(\omega^{\prime}\right)=\frac{\omega^{\prime}(1-\beta \cos \theta)}{1+\beta}, \\
\omega_{\mathrm{T}}^{\prime}(\omega)=\frac{\omega(1+\beta)}{1-\beta \cos \theta} .
\end{gathered}
$$

For linear Compton scattering the scattering cross section, $d \sigma / d \Omega$, is 


$$
\begin{gathered}
\frac{d \sigma}{d \Omega}=\frac{r_{e}^{2}}{2 \gamma^{2}(1+\beta)^{2}}\left(\frac{\omega^{\prime}}{\omega}\right)^{2} \frac{d \tilde{\sigma}}{d \Omega}, \\
\frac{d \tilde{\sigma}}{d \Omega}=\frac{\omega^{\prime}(1-\beta \cos \theta)}{\omega(1+\beta)}+\frac{\omega(1+\beta)}{\omega^{\prime}(1-\beta \cos \theta)}-2 \\
+2 \frac{(\cos \theta-\beta)^{2}}{(1-\beta \cos \theta)^{2}} \cos ^{2} \phi+2 \sin ^{2} \phi,
\end{gathered}
$$

where $r_{e}$ is the classical electron radius. The cross section $d \sigma / d \Omega$ and the bracket $\left[\left(\omega^{\prime} / \omega\right)\left(d \omega / d \omega^{\prime}\right)\right]$ in Eq. (3) are valid in the Compton regime where electron recoil is accounted for. The Thomson limit is recovered upon simply setting $\hbar=0$.

To understand the nonlinear generalizations, it is important to separate the total cross section into scattering with polarization perpendicular to the plane of scattering and with polarization parallel to the plane of scattering. The separation of the cross section is

$\frac{d \tilde{\sigma}_{\perp}}{d \Omega}=\frac{\omega^{\prime}(1-\beta \cos \theta)}{2 \omega(1+\beta)}+\frac{\omega(1+\beta)}{2 \omega^{\prime}(1-\beta \cos \theta)}-1+2 \sin ^{2} \phi$,

$$
\begin{aligned}
\frac{d \tilde{\sigma}_{\|}}{d \Omega}= & \frac{\omega^{\prime}(1-\beta \cos \theta)}{2 \omega(1+\beta)}+\frac{\omega(1+\beta)}{2 \omega^{\prime}(1-\beta \cos \theta)}-1 \\
& +2 \frac{(\cos \theta-\beta)^{2}}{(1-\beta \cos \theta)^{2}} \cos ^{2} \phi
\end{aligned}
$$

The sole remaining term in Eq. (3) for which Compton generalization is sought is the laser field strength $\tilde{E}(k)$. The key idea underpinning our new model is to complete the generalization of the radiation spectrum given in Eq. (3) to the nonlinear Compton regime by approximating $\tilde{E}(k)$ with the nonlinear analog of the effective frequency content from the Thomson nonlinear model $[5,12]$, but with the Compton wave number $k$. Incorporating the nonlinear generalization, the expressions to calculate are

$$
\begin{gathered}
\frac{d^{2} E_{\perp}}{d \omega^{\prime} d \Omega}=\frac{e^{2}}{64 \pi^{3} \epsilon_{0} c^{3}} \omega^{\prime 2} \tilde{D}_{\perp}\left(k^{\prime}\right)\left[\frac{\omega^{\prime}}{\omega} \frac{d \omega}{d \omega^{\prime}}\right], \\
\frac{d^{2} E_{\|}}{d \omega^{\prime} d \Omega}=\frac{e^{2}}{64 \pi^{3} \epsilon_{0} c^{3}} \omega^{\prime 2} \tilde{D}_{\|}\left(k^{\prime}\right)\left[\frac{\omega^{\prime}}{\omega} \frac{d \omega}{d \omega^{\prime}}\right],
\end{gathered}
$$

where the effective frequency content

$$
\begin{aligned}
\tilde{D}_{\perp}\left(k^{\prime}\right)= & \left|\tilde{D}_{x}\left(k^{\prime}\right)\right|^{2}\left(\frac{\omega^{\prime}(1-\beta \cos \theta)}{2 \omega(1+\beta)}+\frac{\omega(1+\beta)}{2 \omega^{\prime}(1-\beta \cos \theta)}-1\right) \\
& +2\left|\tilde{D}_{x}\left(k^{\prime}\right)\right|^{2} \sin ^{2} \phi \\
\tilde{D}_{\|}\left(k^{\prime}\right)= & \left|\tilde{D}_{x}\left(k^{\prime}\right)\right|^{2}\left(\frac{\omega^{\prime}(1-\beta \cos \theta)}{2 \omega(1+\beta)}+\frac{\omega(1+\beta)}{2 \omega^{\prime}(1-\beta \cos \theta)}-1\right) \\
& +2\left|\tilde{D}_{x}\left(k^{\prime}\right) \frac{(\cos \theta-\beta)}{(1-\beta \cos \theta)} \cos \phi+\tilde{D}_{z}\left(k^{\prime}\right) \sin \theta\right|^{2}
\end{aligned}
$$

is composed of form functions $\tilde{D}_{x, z}(k)$ [12],

$$
\tilde{D}_{x, z}\left(k^{\prime}\right)=c_{x, z} \int_{-\infty}^{\infty} d \xi \tilde{A}^{1,2}(\xi) e^{i F(\xi)},
$$

with $c_{x}=1 /[\gamma(1+\beta)]$ and $c_{z}=1 /\left[2 \gamma^{2}(1+\beta)(1-\beta \cos \theta)\right]$,

$$
\begin{aligned}
F(\xi)= & k^{\prime} \frac{\omega_{\mathrm{T}}^{\prime}}{\omega^{\prime}}\left(\frac{\xi(1-\beta \cos \theta)}{1+\beta}-\frac{\sin \theta \cos \phi}{\gamma(1+\beta)} \int_{-\infty}^{\xi} \tilde{A}\left(\xi^{\prime}\right) d \xi^{\prime}\right. \\
& \left.+\frac{1+\cos \theta}{2 \gamma^{2}(1+\beta)^{2}} \int_{-\infty}^{\xi} \tilde{A}^{2}\left(\xi^{\prime}\right) d \xi^{\prime}\right),
\end{aligned}
$$

and $k^{\prime}=\omega^{\prime}(\omega) / c$ given in Eq. (6). The expressions in Eqs. (12) and (13) provide a general model for computation of scattered spectra in the nonlinear Compton regime in which electron recoil is properly accounted for. It subsumes two regimes we developed earlier: nonlinear Thomson and linear Compton. The nonlinear Thomson limit developed in Ref. [12] is recovered upon setting $\hbar=0$ : the term in parentheses in Eq. (13) then vanishes. The linear Compton limit reported in Ref. [10] is recovered by taking the $a_{0} \rightarrow 0$ limit, which leads to $\tilde{D}_{z} \rightarrow 0$.

The form functions $\tilde{D}_{x}(k)$ and $\tilde{D}_{z}(k)$ share the same phase behavior, so any combination of these two terms will be maximized around the same frequency, thereby leading to the derivation of the exact optimal laser FM function.

It has been observed experimentally that the shapes of individual harmonics change with the collision angle between electron and laser beams [14,15]. The model presented here is for a head-on collision of the beams. For this fixed collision angle, the spectral shapes of all harmonics are captured by our model.

Our model is semiclassical by design: a nonlinear quantum frequency correction due to recoil is introduced into a classical model. While other semiclassical calculations may be of value to research within the nonlinear regime of Thomson/Compton scattering, the work presented here has a unique combination of four critically important features: (1) this novel analysis accesses the nonlinear Compton regime; (2) the field of a linearly polarized incident laser pulse is generalized for any envelope shape; (3) the model features an arbitrary FM function within the phase argument; (4) this computational model allows for 3D modeling of the incident wave pulse to 
simulate photon-electron collisions beyond the plane-wave approximation, as described in Ref. [16]. Because of this array of functionality, the work presented here stands out amongst other calculations. CAIN [17], for example, simulates scattering events within the nonlinear Thomson regime, but it cannot account for a chirped laser pulse [16]. CAIN also does not account for the Compton recoil in the scattering electron. Rykovanov et al. [18] presented an FM compensation for a circularly polarized incident laser pulse derived from classical model within the Thomson limit. This chirping correction is a specific application of a broadly generalized FM solution [5].

Other semiclassical calculations are able to provide the exact solution to the nonlinear Compton scattered spectrum by using the Baïer-Katkov (BK) approximation [19] or the Wentzel-Kramers-Brillouin (WKB) model [20,21]. These calculations are able to access more of the nonlinear Compton regime, but they are limited, however, to the plane-wave approximation. These calculations also do not address the implementation of FM to correct ponderomotive broadening. Moreover, these broader nonlinear Compton calculations have yet to be implemented into a functioning computational simulation.

Our semiclassical approach is contrasted with the fully quantum model in Refs. [8,22]. The quantum model is derived from nonperturbative QED utilizing Volkov states. As will be shown below, the two approaches produce equivalent exact chirping prescriptions because the laser phase correction needed for monochromatic emission is fully captured by nonlinear quantum frequency correction.

\section{HIGHER-ORDER HARMONICS IN NONLINEAR COMPTON REGIME}

The form functions $\tilde{D}_{x, z}$ in the two regimes only differ by their arguments: in the Compton regime, the argument is $k=\omega / c$, with $\omega$ given in Eq. (5) and in the Thomson regime, it is $k_{\mathrm{T}}=\omega_{\mathrm{T}} / c$, with $\omega_{\mathrm{T}}$ given in Eq. (7). Therefore, the shapes of $\tilde{D}_{x, z}$ in the Compton regime are identical to those in the Thomson regime, and their locations are related to by a nonlinear scaling of their argument:

$$
k=\frac{\omega\left(\omega^{\prime}\right)}{c}=\frac{\omega_{\mathrm{T}}\left(\omega^{\prime} \frac{1+\beta}{1+\beta-\frac{1 \omega^{\prime}}{\gamma m_{e} c^{2}}(1+\cos \theta)}\right)}{c} .
$$

This scaling means that, when the Compton spectrum is plotted as a function of the scattered frequency $\omega^{\prime}$, it will experience the nonlinear shift from its Thomson spectrum by a factor of

$$
p \equiv \frac{\omega_{\mathrm{T}}^{\prime}}{\omega^{\prime}}=\frac{1-\beta \cos \theta+\frac{\hbar \omega^{\prime}}{\gamma m_{e} c^{2}}(1+\cos \theta)}{1-\beta \cos \theta} .
$$

It is also equal to

$$
p=\frac{\omega_{\mathrm{T}}}{\omega}=\frac{1+\beta}{1+\beta-\frac{\hbar \omega}{\gamma m_{e} c^{2}}(1+\cos \theta)} .
$$

In the backscattered case $(\theta=0)$, for the first harmonic the shift reduces to the familiar

$$
p_{1}=\frac{1}{1+X}
$$

from Eq. (1). This nonlinear shift means that the higherorder harmonics in the Compton regime are no longer evenly spaced, as they were in the Thomson regime: $\tilde{D}_{x}(k)$ at odd and $\tilde{D}_{z}(k)$ at even multiples of the main frequency. Instead, the spacing between consecutive harmonics reduces with the order of the harmonics.

Putting together the shift due to the electron recoil in the Compton regime with the nonlinear shift due to the high laser field regime $\left(a_{0} \gg 0\right)$, the $n$th harmonic of the scattered radiation will be located at

$$
\begin{aligned}
E_{n}^{\prime} \equiv \hbar \omega_{n}^{\prime} & =\frac{n \hbar \tilde{\omega}^{\prime}}{\left(1+a_{0}^{2} / 2\right)} \frac{1+\beta}{1+\beta+\frac{n \hbar \tilde{\omega}^{\prime}}{\left(1+a_{0}^{2} / 2\right) \gamma m_{e} c^{2}}(1+\cos \theta)} \\
& =\frac{n \tilde{E}^{\prime}}{\left(1+a_{0}^{2} / 2\right)} \frac{1+\beta}{1+\beta+\frac{n \tilde{E}^{\prime}}{\left(1+a_{0}^{2} / 2\right) \gamma m_{e} c^{2}}(1+\cos \theta)},
\end{aligned}
$$

where $\tilde{E}^{\prime} \equiv \hbar \tilde{\omega}^{\prime}=\hbar \omega_{0}(1+\beta) /(1-\beta \cos \theta), \omega_{0}=2 \pi c / \lambda$ and $E_{\mathrm{L}}=\hbar \omega_{0}$. In the backscattered case $(\theta=0)$, $\tilde{E}^{\prime}=4 \gamma^{2} E_{\mathrm{L}}$, so harmonics will be located at

$$
\begin{aligned}
E_{n}^{\prime}=\hbar \omega_{n}^{\prime} & =\frac{4 n \gamma^{2} E_{\mathrm{L}}}{\left(1+a_{0}^{2} / 2\right)\left[1+n X /\left(1+a_{0}^{2} / 2\right)\right]} \\
& =\frac{4 n \gamma^{2} E_{\mathrm{L}}}{1+a_{0}^{2} / 2+n X} .
\end{aligned}
$$

The well-known shifts in the two regimes previously investigated, $1 /\left(1+a_{0}^{2} / 2\right)$ in the nonlinear Thomson $(X \approx 0)[12,13]$ and $1 /(1+X)$ in the linear Compton $\left(a_{0} \approx 0\right)$ [11] regimes, are easily recovered from Eq. (21).

Further exploring Eq. (21), we note that the bunched up harmonics will converge to

$$
\lim _{n \rightarrow \infty} \frac{E_{n}^{\prime}}{\tilde{E}^{\prime}}=\lim _{n \rightarrow \infty} \frac{E_{n}^{\prime}}{4 \gamma^{2} E_{\mathrm{L}}}=\frac{1}{X} .
$$

This should come as no surprise, because the energy that a photon gains during a collision with an electron cannot exceed the electron's energy, i.e., $\hbar \omega^{\prime} \leq E_{e}$, or $\hbar \omega^{\prime} / E_{e} \leq 1$.

The nonlinear relationship between the Compton and Thomson scattered frequencies, given in Eqs. (6) and (8), is 

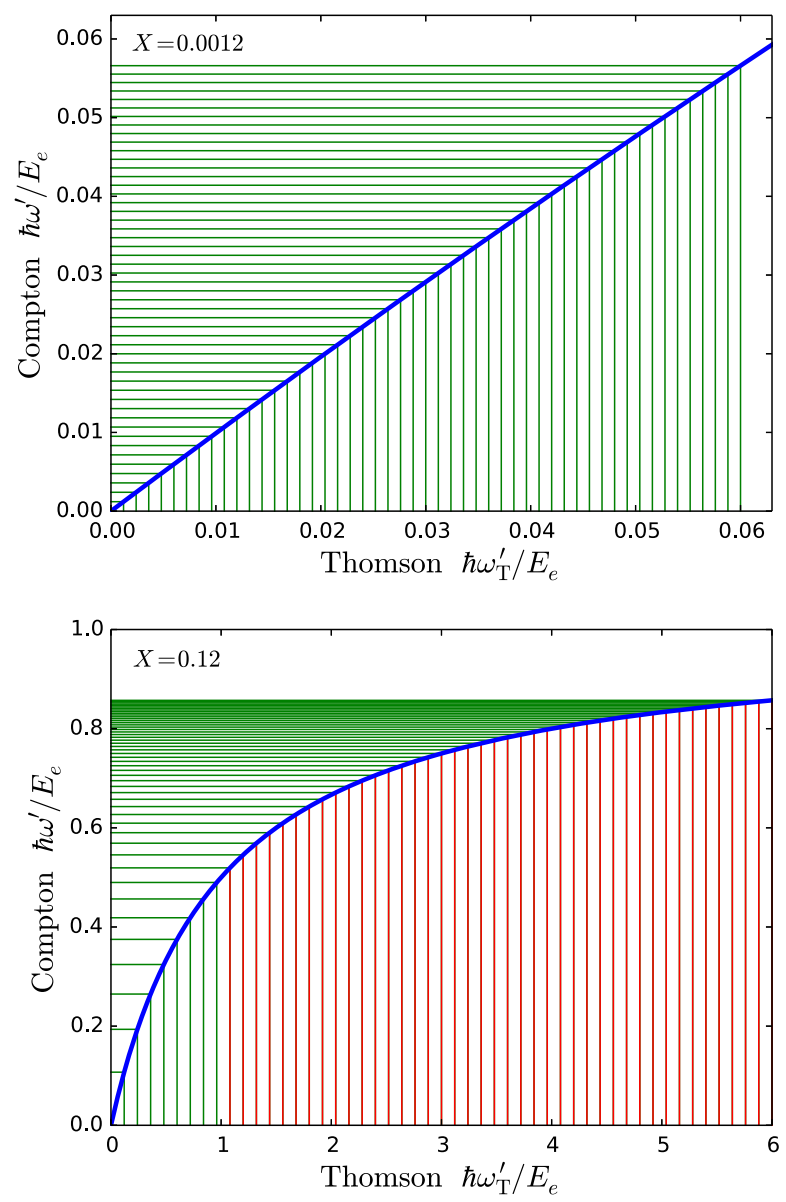

FIG. 1. Relationship between the scattered frequencies in Compton and Thomson regime (blue line), including the first 50 harmonics for $X=0.0012$ (top panel) and $X=0.12$ (bottom panel). Harmonics in the Thomson regime which are at higher energies than the rms electron energy $\hbar \omega_{\mathrm{T}}^{\prime}>E_{e}$ are nonphysical and are marked in red. The physical harmonics are shown in green.

shown in Fig. 1. The bunching of the higher-order harmonics in the Compton regime with non-negligible recoil parameter $X$ and its slow convergence toward $1 / X$ is shown in the bottom panel of Fig. 1 and the bottom right panel of Fig. 2. Because of the precipitous drop in power as the order of the harmonic increases, as well as the very slow approach to the limit, convergence to $1 / X$ is not observable in the scattered spectra within 30 orders of magnitude. This nonlinear spacing of the higher harmonics in the Compton regime was also reported in Ref. [22] (see their Fig. 6).

\section{DERIVATION OF THE OPTIMAL FREQUENCY MODULATION IN THE COMPTON REGIME}

We use our new, nonlinear Compton model developed in Eqs. (12) and (13) to derive a general expression for the optimal FM for an arbitrary optimization angle $\theta_{0}$ in spherical coordinates. This angle is not to be confused with the observation angle $\theta$ at which the radiation spectrum is computed.

First, we allow for FM by representing the laser pulse by a normalized vector potential $\widetilde{A}(\xi)=$ $a(\xi) \cos \left[2 \pi \xi f(\xi) / \lambda_{0}\right]$, where $f(\xi)$ is the FM function. Let us seek two different FM functions, $f_{x}(\xi)$ and $f_{z}(\xi)$ which maximize $\tilde{D}_{x}(k)$ and $\tilde{D}_{z}(k)$, respectively. Maximizing the peaks of the effective frequency content functions $\tilde{D}_{x}(k)$ and $\tilde{D}_{z}(k)$ also minimizes their bandwidth. Using the relations

$$
\begin{aligned}
\cos \alpha & =\frac{1}{2}\left(e^{i \alpha}+e^{-i \alpha}\right), \\
\cos ^{2} \alpha & =\frac{1}{2}(1+\cos 2 \alpha),
\end{aligned}
$$

the expressions for $\tilde{D}_{x}(k)$ and $\tilde{D}_{z}(k)$ from Eq. (14) become

$$
\begin{aligned}
\tilde{D}_{x}(k)= & \frac{c_{x}}{2} \int_{-\infty}^{\infty} d \xi a(\xi) e^{i\left[F(\xi)+\frac{2 \pi \xi \xi f_{x}(\xi)}{\lambda}\right]} \\
& +\frac{c_{x}}{2} \int_{-\infty}^{\infty} d \xi a(\xi) e^{i\left[F(\xi)-\frac{2 \pi \xi f_{x}(\xi)}{\lambda}\right]},
\end{aligned}
$$

and

$$
\begin{aligned}
\tilde{D}_{z}(k)= & \frac{c_{z}}{2} \int_{-\infty}^{\infty} d \xi a^{2}(\xi) e^{i F(\xi)}+\frac{c_{z}}{4} \int_{-\infty}^{\infty} d \xi a^{2}(\xi) e^{i\left[F(\xi)+\frac{4 \pi \xi f_{z}(\xi)}{\lambda}\right]} \\
& +\frac{c_{z}}{4} \int_{-\infty}^{\infty} d \xi a^{2}(\xi) e^{i\left[F(\xi)-\frac{4 \pi \xi f_{z}(\xi)}{\lambda}\right]}
\end{aligned}
$$

As in the original derivation [5], we use the stationary phase method to find the functional forms of $f_{x, z}(\xi)$ for which the phases given in Eqs. (25) and (26) are constant. For both expressions in Eqs. (25) and (26), only the phase in the last term is not monotonically increasing and can possibly be constant. Therefore, the quantities $\tilde{D}_{x}(k)$ and $\tilde{D}_{z}(k)$ will be maximized when

$$
\begin{aligned}
& \frac{d}{d \xi}\left(F(\xi)-\frac{2 \pi \xi f_{x}(\xi)}{\lambda}\right)=0, \\
& \frac{d}{d \xi}\left(F(\xi)-\frac{4 \pi \xi f_{z}(\xi)}{\lambda}\right)=0 .
\end{aligned}
$$

To solve these equations with $F(\xi)$ given in Eq. (15), we use approximations

$$
\begin{aligned}
& \int_{-\infty}^{\xi} \tilde{A}^{2}\left(\xi^{\prime}\right) d \xi^{\prime} \\
& \approx \frac{1}{2} \int_{-\infty}^{\xi} a^{2}\left(\xi^{\prime}\right) d \xi^{\prime}+\frac{1}{2} \frac{\lambda}{2 \pi \xi f(\xi)} a^{2}(\xi) \cos \left(\frac{2 \pi \xi f(\xi)}{\lambda}\right) \\
& \times \sin \left(\frac{2 \pi \xi f(\xi)}{\lambda}\right)
\end{aligned}
$$



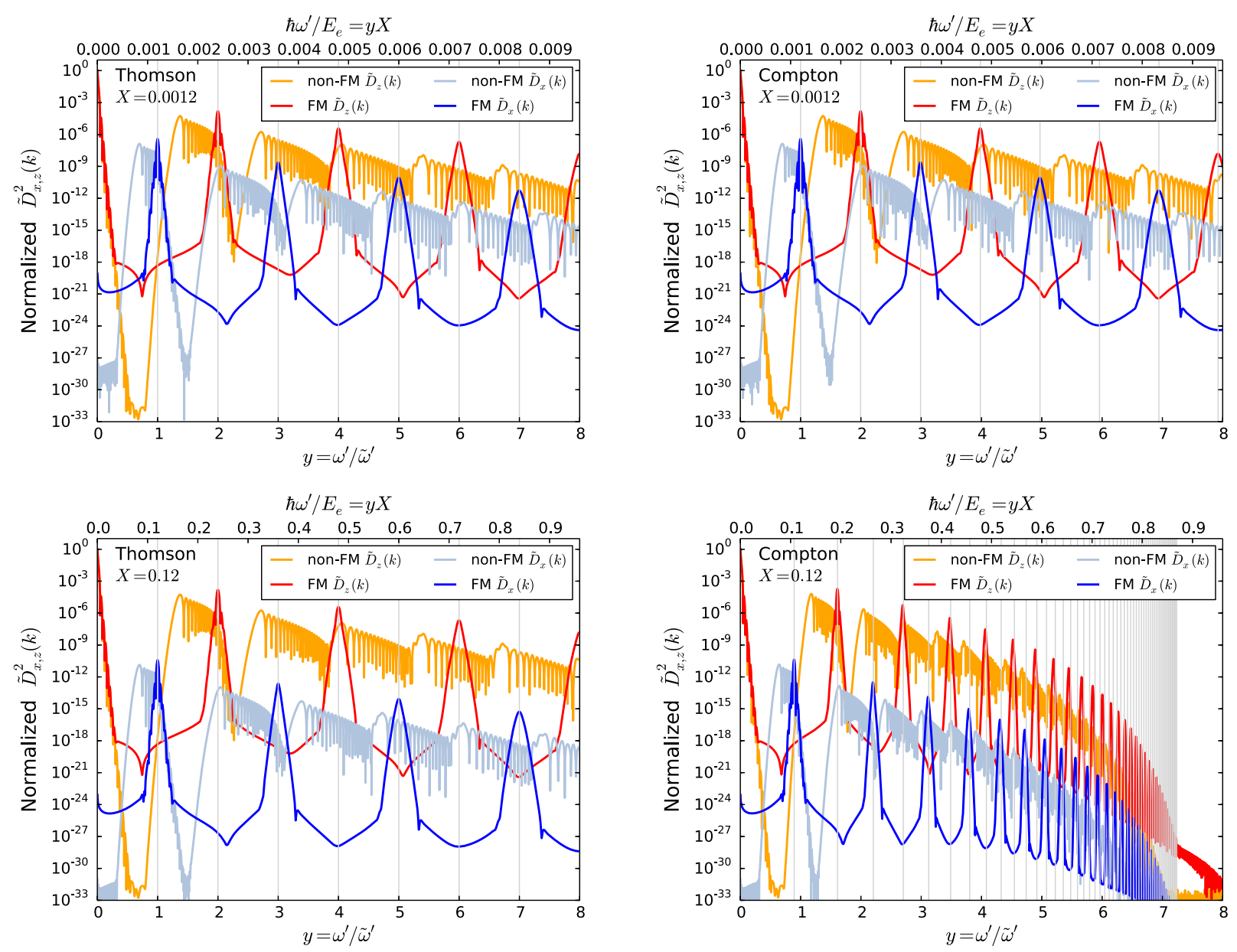

FIG. 2. Frequency content functions $\tilde{D}_{x}(k)$ and $\tilde{D}_{z}(k)$ with and without optimal FM for backscattering $(\theta=0)$, normalized to the maximum value of $\tilde{D}_{z}(k)$. For the FM spectra, $f_{0}=1$, so that the FM harmonics in the Thomson regime occur at integer values of $y \equiv \omega^{\prime} / \tilde{\omega}^{\prime}$; the deviation from their integer values in the Compton regime is caused by the shift due to parameter $X$. For both $\tilde{D}_{x}(k)$ and $\tilde{D}_{z}(k)$, the same FM function given in Eq. (35) is used. The calculation is for the head-on scattering of a linearly polarized laser with the wavelength of $\lambda=800 \mathrm{~nm}$ and the pulse duration of $21 \mathrm{fs}$ and $a_{0}=1$ on an electron beam with initial energy $51 \mathrm{MeV}(X=0.0012)$ (top row) and $5.1 \mathrm{GeV}(X=0.12)$ (bottom row). The right column represents solutions in the Compton regime, captured in Eqs. (25) and (26). The left column shows the Thomson limit, when $\hbar=0$ in Eqs. (25) and (26). Vertical gray lines denote the locations of the harmonics given in Eq. (38). The energy that a scattered photon gains during a collision with an electron cannot exceed the electron's energy, so only scattered energies for which $h \omega^{\prime} / E_{e}=y X \leq 1$ are physical. A Gaussian laser envelope $a_{\mathrm{G}}(\xi)=a_{0} \exp \left\{-\xi^{2} /\left[2(\bar{\sigma} \lambda)^{2}\right]\right\}$ is used, with $\bar{\sigma}$ the normalized laser pulse length $\sigma=\bar{\sigma} \lambda$.

and

$$
\begin{aligned}
\int_{-\infty}^{\xi} \tilde{A}\left(\xi^{\prime}\right) d \xi^{\prime} & =\int_{-\infty}^{\xi} a\left(\xi^{\prime}\right) \cos \left(\frac{2 \pi \xi^{\prime} f\left(\xi^{\prime}\right)}{\lambda}\right) d \xi \\
& \approx a(\xi) \frac{\lambda \sin \left(\frac{2 \pi \xi f(\xi)}{\lambda}\right)}{2 \pi\left[f(\xi)+\xi f^{\prime}(\xi)\right]} \approx a(\xi) \frac{\lambda \sin \left(\frac{2 \pi \xi f(\xi)}{\lambda}\right)}{2 \pi f(\xi)} \\
& =a(\xi) \xi \frac{\sin \left(\frac{2 \pi \xi f(\xi)}{\lambda}\right)}{\frac{2 \pi \xi f(\xi)}{\lambda}}=a(\xi) \xi j_{0}\left(\frac{2 \pi \xi f(\xi)}{\lambda}\right)
\end{aligned}
$$

where $j_{0}(x)$ is the spherical Bessel function. Here we invoked the slowly varying envelope approximation twice. Near the resonance, $k^{\prime}=s(1+\beta)^{2} \gamma^{2}(2 \pi / \lambda)=$ $s(1+\beta) 2 \pi /[\lambda(1-\beta)]$, where $s \equiv 1 /\left(1+a_{0}^{2} / 2\right)$ is the nonlinear shift. Then Eqs. (27a) and (27b) simplify to

$$
\frac{d}{d \xi} \Phi_{x, z}=0
$$

where

$$
\begin{aligned}
\Phi_{x}= & \frac{s(1-\beta \cos \theta) \xi}{(1-\beta)}+s \sin \theta \cos \phi a(\xi) \xi j_{0}\left(\frac{2 \pi \xi f_{x}(\xi)}{\lambda}\right) \\
& +\frac{s(1+\cos \theta)}{4} \int_{-\infty}^{\xi} a^{2}\left(\xi^{\prime}\right) d \xi^{\prime}-\xi f_{x}(\xi),
\end{aligned}
$$




$$
\begin{aligned}
\Phi_{z}= & \frac{s(1-\beta \cos \theta) \xi}{(1-\beta)}+s \sin \theta \cos \phi a(\xi) \xi j_{0}\left(\frac{2 \pi \xi f_{z}(\xi)}{\lambda}\right) \\
& +\frac{s(1+\cos \theta)}{4} \int_{-\infty}^{\xi} a^{2}\left(\xi^{\prime}\right) d \xi^{\prime}-2 \xi f_{z}(\xi) .
\end{aligned}
$$

We use the Leibniz integral rule to find

$$
\frac{d}{d \xi} \int_{-\infty}^{\xi} \tilde{A}\left(\xi^{\prime}\right) d \xi^{\prime}=\tilde{A}(\xi)=a(\xi) \cos \left(\frac{2 \pi \xi f(\xi)}{\lambda}\right)
$$

Solving for $f(\xi)$, we finally arrive at the optimal FM function for maximizing $\tilde{D}_{x}(k)$ :

$$
\begin{aligned}
f_{x}(\xi)= & f_{0}\left[\frac{1-\beta \cos \theta_{0}}{1-\beta}+\sin \theta_{0} \cos \phi_{0} a(\xi) \cos \left(\frac{2 \pi \xi f_{x}(\xi)}{\lambda}\right)\right. \\
& \left.+\frac{1+\cos \theta_{0}}{4 \xi} \int_{0}^{\xi} a^{2}\left(\xi^{\prime}\right) d \xi^{\prime}\right],
\end{aligned}
$$

and for maximizing $\tilde{D}_{z}(k)$

$$
f_{z}(\xi)=\frac{1}{2} f_{x}(\xi)
$$

$\theta_{0}$ is the angle for which the FM is exact and $f_{0}$ is a normalization constant such that $f_{0} \omega_{0}$ is the laser frequency at the center of the pulse. For relativistic electron beams $(\gamma>10)$ and at an observation angle with significant flux $\left(\theta_{0} \leq 1 / \gamma\right)$, the second term in Eq. (33) is negligible compared to the other two terms, leading to the expression for the optimal FM:

$f_{x}(\xi)=f_{0}\left(\frac{1-\beta \cos \theta_{0}}{1-\beta}+\frac{1+\cos \theta_{0}}{4 \xi} \int_{0}^{\xi} a^{2}\left(\xi^{\prime}\right) d \xi^{\prime}\right)$.

In the original derivation of the optimal FM for a 1D plane wave [5], $f_{0}=s=1 /\left(1+a_{0}^{2} / 2\right)$ was adopted, such that $f(0)=1$, while consequent studies used $f_{0}$ such that $f( \pm \infty)=1[7,8,18,23]$. The exact FM functions for three different shapes of the laser envelope-Gaussian $a_{\mathrm{G}}$, hyperbolic secant $a_{\mathrm{S}}$ and Lorentzian $a_{\mathrm{L}}$ :

$$
\begin{aligned}
& a_{\mathrm{G}}(\xi)=a_{0} \exp \left\{-\xi^{2} /\left[2(\bar{\sigma} \lambda)^{2}\right]\right\} \\
& a_{\mathrm{S}}(\xi)=a_{0} \operatorname{sech}(\bar{\sigma} \xi / \lambda) \\
& a_{\mathrm{L}}(\xi)=a_{0} \bar{\sigma} /[(\xi / \lambda)+\bar{\sigma}]
\end{aligned}
$$

where $\sigma=\bar{\sigma} \lambda$ is the characteristic length of the laser pulse, are computed from the Eq. (35) to be

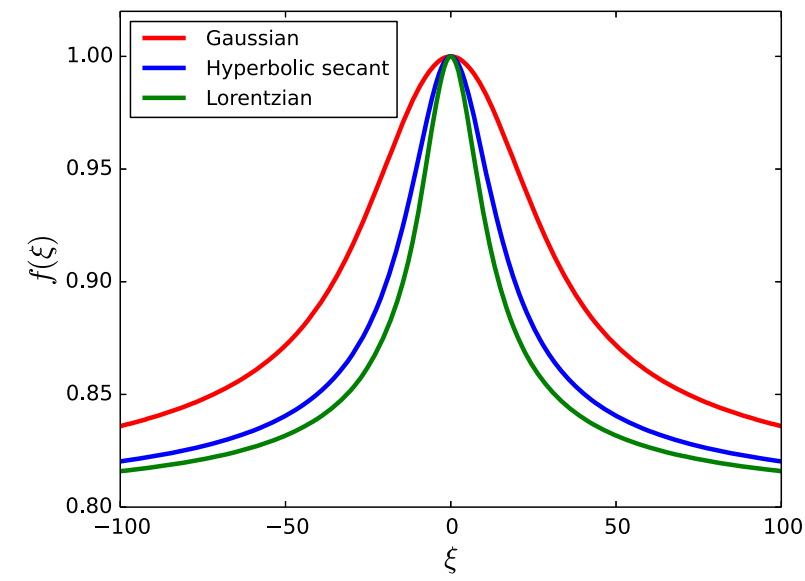

FIG. 3. Exact FM functions for a Gaussian (red line); hyperbolic secant (blue line) and Lorentzian (green line). Plots are for the head-on scattering of a linearly polarized laser with pulse duration of $\bar{\sigma}=20.25$ and $a_{0}=\sqrt{2} / 2=0.707$ and $\theta_{0}=0$. Here the normalization $f_{0}=1 /\left(1+a_{0}^{2} / 2\right)$ is adopted, so that $f(0)=1$, and $f( \pm \infty)=f_{0}$. In this case $f_{0}=0.8$. The resulting spectra for $E=163 \mathrm{MeV}$ electron beam and $\lambda=1 \mu \mathrm{m}$ are shown in Fig. 3 of Ref. [24].

$$
\begin{aligned}
f_{\mathrm{G}}(\xi) & =f_{0}\left(\frac{1-\beta \cos \theta_{0}}{1-\beta}+\frac{\left(1+\cos \theta_{0}\right) \sqrt{\pi} \sigma a_{0}^{2}}{8 \xi} \operatorname{erf}(\xi / \sigma)\right) \\
f_{\mathrm{S}}(\xi) & =f_{0}\left(\frac{1-\beta \cos \theta_{0}}{1-\beta}+\frac{\left(1+\cos \theta_{0}\right) a_{0}^{2}}{4 \xi \sigma} \tanh (\sigma \xi)\right) \\
f_{\mathrm{L}}(\xi) & =f_{0}\left[\frac{1-\beta \cos \theta_{0}}{1-\beta}+\frac{\left(1+\cos \theta_{0}\right) \sqrt{\bar{\sigma}} a_{0}^{2}}{8 \sqrt{2} \xi}\right. \\
& \left.\times\left(\frac{\sqrt{2 \bar{\sigma} \xi}}{\bar{\sigma}+2 \xi^{2}}+\tan ^{-1} \frac{\sqrt{2} \xi}{\sqrt{\bar{\sigma}}}\right)\right] .
\end{aligned}
$$

These FM are shown in Fig. 3.

The multiplicative constant $f_{0}$ in the FM function in Eq. (35) shifts the scattered energy spectrum by a factor $f_{0}$, without changing its shape. Therefore, Eq. (34) suggests that the optimal FM function $f_{x}(\xi)$ given in Eq. (35) not only perfectly restores the bandwidth of $\tilde{D}_{x}(k)$ at some frequency $\omega_{\max }^{\prime}$, but also simultaneously restores the bandwidth for $\tilde{D}_{z}(k)$ at $2 \omega_{\max }^{\prime}$. That is why $\tilde{D}_{x}(k)$, which is the only form factor contributing to the perfectly backscattered $(\theta=0)$ spectrum, only has odd harmonics, and $\tilde{D}_{z}(k)$ only even. Because FM shifts the corrected spectrum by $f_{0}$, the harmonics will be located at

$$
E_{n}^{\prime}=\hbar \omega_{n}^{\prime}=\frac{4 n \gamma^{2} E_{\mathrm{L}} f_{0}}{\left(1+n X f_{0}\right)}=\frac{4 n \gamma^{2} E_{\mathrm{L}}}{\left(1 / f_{0}+n X\right)} .
$$

Figure 2 shows the quantities $\tilde{D}_{x}^{2}(k)$ and $\tilde{D}_{z}^{2}(k)$ with and without optimal FM given in Eq. (35) with $f_{0}=1$ for backscattering $(\theta=0)$, normalized to the maximum value of $\tilde{D}_{z}(k)$. The left column shows the Thomson, no-recoil limit (when $\hbar=0$ in $k$ ) of the expressions in Eqs. (25) and 
(26) for two different electron energies, $E=51 \mathrm{MeV}$ $(X=0.0012)$ (top) and $E=5.1 \mathrm{GeV}(X=0.12)$ (bottom panel). The right column shows the full Compton solution in Eqs. (25) and (26), for the same energies.

Two striking features of the scattered radiation in the Compton regime are evident from Fig. 2. First, the primary peak as well as all higher-order harmonics are perfectly compensated with the same frequency modulation function. Second, the higher-order harmonics are bunched closer together, with their spacing decreasing as the harmonic order increases. The bunching becomes more pronounced as the recoil parameter $X$ grows [22].

For backscattering, $\theta=0$, so the constants multiplying the integrands in Eqs. (25) and (26) become $c_{x}=1 /[\gamma(1+\beta)]$ and $c_{z}=1 / 2$. In the Thomson regime, $\hbar=0$, so $k^{\prime}=$ $k(1+\beta) /(1-\beta \cos \theta)$. Therefore, in the case of backscattering, $k^{\prime}=k(1+\beta) /(1-\beta)$ and Eq. (15) reduces to

$$
F(\xi)=k\left(\xi+\int_{-\infty}^{\xi} \tilde{A}^{2}\left(\xi^{\prime}\right) d \xi^{\prime}\right),
$$

which means that the integrands themselves are independent of energy. $\tilde{D}_{x}(k)$ scales as $1 / \gamma$, and $\tilde{D}_{z}(k)$ is independent of $\gamma$, because of the constants $c_{x}=1 /[\gamma(1+\beta)]$ and $c_{z}=1 / 2$, respectively. This is readily observed as we compare Thomson solutions shown in the left column of Fig. 2.

For small values of the electron recoil parameter $X$, such as those in the top row of Fig. 2, the quantities $\tilde{D}_{x}(k)$ and $\tilde{D}_{z}(k)$ in Thomson and Compton regimes are indistinguishable, as expected. With increasing values of $X$, the properly accounting for electron recoil changes spectra by reducing the spacing between the consecutive harmonics. The bunched up harmonics eventually converge to $y=1 / X$, as predicted by Eq. (22).

The peak of the function $\tilde{D}_{z}(k)$ at the origin does not directly translate to emission. As can be seen from Eq. (12), the emission $d^{2} E / d \omega^{\prime} d \Omega$ is also affected by the scattered frequency $\omega^{\prime}$ and the cross section $d \tilde{\sigma} / d \Omega$.

$\tilde{D}_{x}(k)$ captures the odd harmonics and $\tilde{D}_{z}(k)$ the even, as shown in Fig. 2. For the perfect backscattering $(\theta=0)$, only the odd harmonics are present, so the spectrum only features a $\tilde{D}_{x}(k)$ component.

The property of the exact FM function to have a maximum in the middle of the pulse and symmetrically drop from there is suggestive of a possible practical realization of the FM. Constructing an FEL oscillator in which the driving beam bunches are long enough that the rf-curvature-related energy spread is appreciable will produce a FM laser pulse [5]. Another promising practical realization may be a sawtooth FM function: linearly chirped up from the front to the middle of the pulse, and then linearly chirped down from the middle to the back of the pulse. Such imperfect, but experimentally realizable FM functions still recover the narrow spectral bandwidth to within a few percent of what is expected with the exact FM functions.

\section{BACKSCATTERED SPECTRA FOR HEAD-ON SCATTERING OFF A GAUSSIAN LASER PULSE}

In the special case of inverse Compton backscattering $(\theta=0)$ of an on-axis electron, and with $f(0)=1$, Eq. (35) reduces to the familiar Eq. (4) in Ref. [5]:

$$
f(\xi)=\frac{1}{1+a_{0}^{2} / 2}\left(1+\frac{1}{2 \xi} \int_{0}^{\xi} a^{2}\left(\xi^{\prime}\right) d \xi^{\prime}\right) .
$$
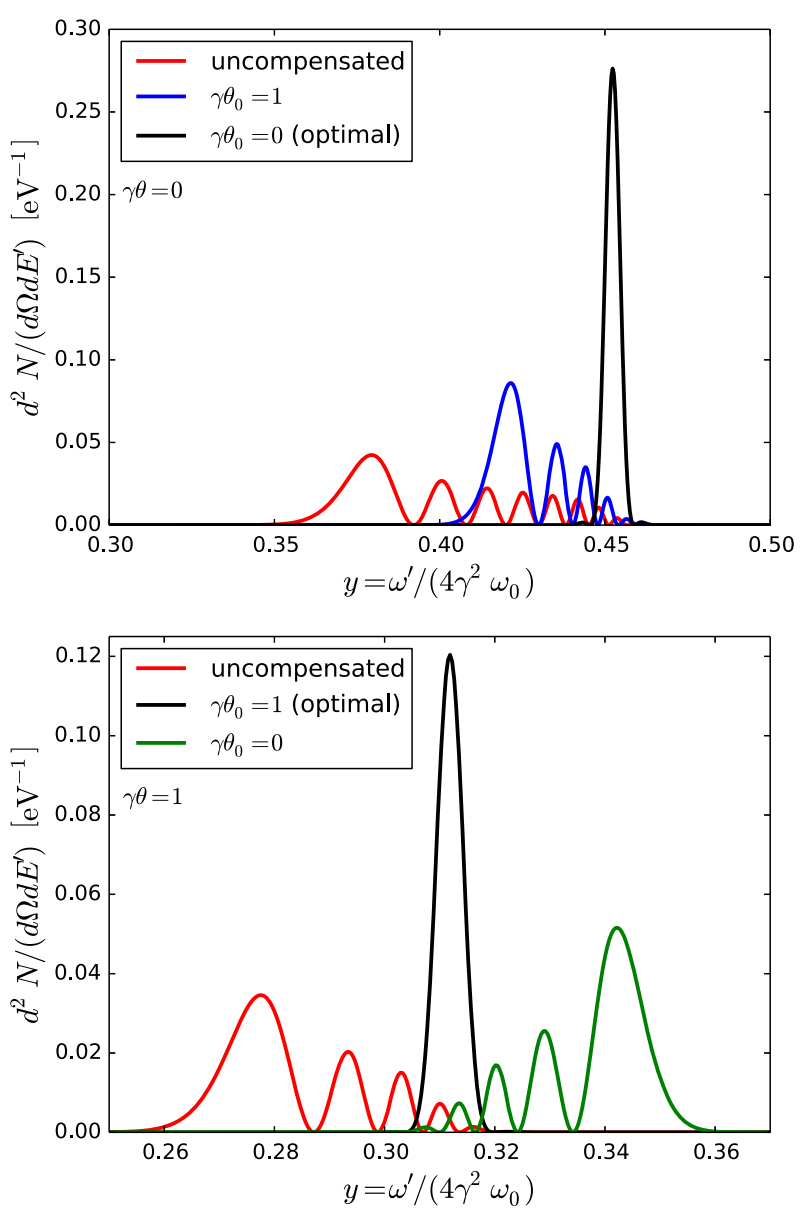

FIG. 4. Top: Radiation spectrum for the compensated nonlinear Compton backscattering $(\theta=0)$. The optimally compensated spectrum shown in black for $\theta_{0}=\theta$ is much narrower than the uncompensated spectrum (red line) and partially compensated spectrum (blue line), where the optimization angle $\theta_{0}$ does not coincide with the scattering angle $\theta$. Bottom: Radiation spectra for the compensated nonlinear Compton scattering at an angle $\theta=1 / \gamma$ and $\phi=\pi / 2$. The optimally compensated spectrum is shown in black for $\theta_{0}=\theta=1 / \gamma$, uncompensated in red and the spectrum for which the optimization angle does not coincide with the scattering angle $\left(\theta_{0}=0\right)$ in green. Top and bottom panels are to be compared with Figs. 2(a) and 2(b) from Ref. [8], respectively. Our spectra assume both positive and negative frequencies exist in the spectra; Ref. [8] assumes positive frequency only and is therefore a factor of 2 higher. Both plots are for the head-on scattering of a linearly polarized $800 \mathrm{~nm}$ laser with pulse duration of 21 fs and $a_{0}=1$ on an electron beam with initial energy $51 \mathrm{GeV}$. A Gaussian laser envelope is used. 
For a Gaussian laser envelope, $a(\xi)=a_{0} \exp \left[-\xi^{2} /\left(2 \sigma^{2}\right)\right]$, with $\sigma$ the longitudinal size of the laser pulse, the optimal FM in Eq. (35) becomes

$f_{g}(\xi)=f_{0}\left[\frac{1-\beta \cos \theta_{0}}{1-\beta}+\frac{\sqrt{\pi} \sigma a_{0}^{2}\left(1+\cos \theta_{0}\right)}{8 \xi} \operatorname{erf}\left(\frac{\xi}{\sigma}\right)\right]$,

where $\operatorname{erf}(x)$ is an error function. To make contact with the optimal FM reported in Ref. [8] for a Gaussian laser pulse, we use their FM form reported in terms of angular frequency $\omega$ :

$$
\omega(\xi)=\omega_{0}\left(1+\frac{1}{1+\gamma^{2} \theta_{0}^{2}} \frac{a_{0}^{2}}{2} g^{2}(\xi)\right),
$$

where $g(\xi)=\exp \left[-\xi^{2} /\left(2 \sigma^{2}\right)\right]$. The corresponding optimal FM $f(\xi)$ is derived by solving

$$
\frac{d}{d \xi}[\xi f(\xi)]=\omega(\xi)
$$

with their normalization constant $f( \pm \infty)=1$. The resulting FM form is

$$
f_{g}(\xi)=1+\frac{1}{1+\gamma^{2} \theta_{0}^{2}} \frac{\sqrt{\pi} \sigma a_{0}^{2}}{4 \xi} \operatorname{erf}\left(\frac{\xi}{\sigma}\right)
$$

which is easily recovered from our Eq. (41) after using a normalization constant $f( \pm \infty)=1$ and a smallangle approximation for $\cos \theta_{0}$, which is well justified because $\theta_{0} \in[0,1 / \gamma]$.

The perfect recovery of the narrow band property of the backscattered radiation by using the optimal FM from Eq. (41) is shown in Fig. 4. Whenever the optimization angle $\theta_{0}$ coincides with the observation angle $\theta$, the recovery of the narrow band property of the scattered radiation using the optimal FM is perfect. However, for any other observation angle $\theta$, the compensation is only partial. The spectra shown in Fig. 4 are a match to the spectra reported in Fig. 2 of Ref. [8], demonstrating the equivalence of their quantum model and our semianalytical model in the case of a Gaussian pulse.

\section{CONCLUSION}

Both the earlier, QED-based [8], and the present, semiclassical derivation of the optimal FM, reveal that its form is the same regardless if the electron recoil is neglected (Thomson regime) or properly accounted for (Compton regime). The optimal FM only compensates the bandwidth of scattered radiation at one scattering angle at a time, and also depends on the electron energy [8]. These features were not appreciated in the original report of the optimal FM [5], because that derivation addressed only backscattered radiation $(\theta=0)$, for which these dependencies are suppressed.

We derived new expressions for radiated spectra in the nonlinear Compton regime, with electron recoil properly accounted for. These include spectra including FM. The theoretical formalism developed here is used to find the locations of all harmonics in the high laser field $\left(a_{0} \gg 0\right)$ regime and at high electron energies $(X \gg 0)$. We predict that they will be nonevenly spaced-redshifted in the nonlinear manner. Exact locations of the main peak and the higher-order harmonics are also presented here.

The optimal FM of the laser pulse counteracts for the deleterious effects of the nonlinear broadening of the radiation spectra in ICS, thereby significantly extending their science reach. The expressions reported here provide the first complete set of optimal FM functions for all regimes of operations of ICS and for all laser shape sizes.

\section{ACKNOWLEDGMENTS}

This paper is authored by Jefferson Science Associates, LLC under U.S. Department of Energy Contract No. DEAC05-06OR23177. B. T. acknowledges the support from the U.S. National Science Foundation Grants No. 1535641 and No. 1847771 . E. J. was supported by the U.S. National Science Foundation Grant No. 1847771. J. M. was supported by the U.S. National Science Foundation Research Experience for Undergraduates at Old Dominion University Grant No. 1659177.

[1] G. A. Krafft and G. Priebe, Rev. Accel. Sci. Techol. 03, 147 (2010).

[2] C. Sun and Y. K. Wu, Phys. Rev. ST Accel. Beams 14, 044701 (2011).

[3] O. Adriani et al., ELI-NP-GBS Technical Design Report, http://arxiv.org/ftp/arxiv/papers/1407/1407.3669.pdf, 2014.

[4] D. Micieli, I. Drebot, A. Bacci, E. Milotti, V. Petrillo, M. Rossetti Conti, A. R. Rossi, E. Tassi, and L. Serafini, Phys. Rev. Accel. Beams 19, 093401 (2016).

[5] B. Terzić, K. Deitrick, A. S. Hofler, and G. A. Krafft, Phys. Rev. Lett. 112, 074801 (2014).

[6] I. Ghebregziabher, B. A. Shadwick, and D. Umstadter, Phys. Rev. Accel. Beams 16, 030705 (2013).

[7] C. Maroli, V. Petrillo, I. Drebot, L. Serafini, B. Terzić, and G. Krafft, J. Appl. Phys. 124, 063105 (2018).

[8] D. Seipt, S. G. Rykovanov, A. Surzhykov, and S. Fritzsche, Phys. Rev. A 91, 033402 (2015).

[9] C. Curatolo, I. Drebot, V. Petrillo, and L. Serafini, Phys. Rev. Accel. Beams 20, 080701 (2017).

[10] G. Krafft, E. Johnson, K. Deitrick, B. Terzić, R. Kelmar, T. Hodges, W. Melnitchouk, and J. Delayen, Phys. Rev. Accel. Beams 19, 121302 (2016).

[11] N. Ranjan, B. Terzić, G. A. Krafft, V. Petrillo, I. Drebot, and L. Serafini, Phys. Rev. Accel. Beams 21, 030701 (2018).

[12] G. A. Krafft, Phys. Rev. Lett. 92, 204802 (2004). 
[13] C. Brau, Phys. Rev. ST Accel. Beams 7, 020701 (2004).

[14] S. Chen, A. Maksimchuk, and D. Umstadter, Nature (London) 396, 653 (1998).

[15] M. Babzien et al., Phys. Rev. Lett. 96, 054802 (2006).

[16] B. Terzić, A. Brown, I. Drebot, T. Hagerman, E. Johnson, G. A. Krafft, C. Maroli, V. Petrillo, and M. Ruijter, Europhys. Lett. 126, 12003 (2019).

[17] P. Chen, G. Horton-Smith, T. Ohgaki, A. W. Weidemann, and K. Yokoya, Nucl. Instrum. Methods Phys. Res., Sect. A 355, 107 (1995).
[18] S. G. Rykovanov, C. G. R. Geddes, C. B. Schroeder, E. Esarey, and W. P. Leemans, Phys. Rev. Accel. Beams 19, 030701 (2016).

[19] X. Artru, Phys. Rev. Accel. Beams 22, 050705 (2019).

[20] A. Di Piazza, Phys. Rev. Lett. 113, 040402 (2014).

[21] A. Di Piazza, Phys. Rev. D 103, 076011 (2021).

[22] D. Seipt and B. Kämpfer, Phys. Rev. A 83, 022101 (2011).

[23] B. Terzić and G. A. Krafft, Phys. Rev. Accel. Beams 19, 098001 (2016).

[24] B. Terzić and C. Reeves, and G. A. Krafft, Phys. Rev. Accel. Beams 19, 044403 (2016). 